\title{
A Case of Uveal Colobomas Showing Marked Left-Right Difference in Diabetic Retinopathy
}

\author{
Takeshi Moriya $^{\mathrm{a}}$ Ryosuke Ochi ${ }^{\mathrm{a}}$ Yukihiro Imagawa ${ }^{\mathrm{a}}$ Bumpei Sato ${ }^{\mathrm{a}}$ \\ Seita Morishita $^{\mathrm{b}}$ Masahiro Tonari $^{\mathrm{b}}$ Masanori Fukumoto $^{\mathrm{b}}$ \\ Hiroyuki Suzuki $^{\mathrm{b}}$ Takatoshi Kobayashi $^{\mathrm{b}}$ Teruyo Kida ${ }^{\mathrm{b}}$ \\ Tsunehiko Ikeda \\ ${ }^{a}$ Department of Ophthalmology, Osaka Kaisei Hospital, Osaka, and ${ }^{b}$ Department of \\ Ophthalmology, Osaka Medical College, Takatsuki, Japan
}

\section{Key Words}

Choroidal coloboma · Diabetic retinopathy · Left-right difference · Optical coherence tomography · Fluorescein fundus angiography

\begin{abstract}
Purpose: Congenital uveal colobomas, including inferior iris and choroidal colobomas, are associated with microcornea and microphthalmia and often show left-right differences (laterality). The purpose of this study was to report a case of choroidal coloboma associated with left-right differences in diabetic retinopathy (DR). Case: This study reports a 59-year-old male with bilateral iris and choroidal colobomas. The colobomatous area in the patient's right eye extended to the macula, and his right eye had been amblyopic since birth. The colobomatous area in his left eye was less extensive and did not involve the macula. Examination of the patient's left eye revealed multiple hemorrhages and hard exudates in the macula due to DR, but examination of his right eye showed almost no changes in DR, thus revealing a marked left-right difference. Optical coherence tomography showed more extensive retinal thinning in the patient's right eye than in his left eye. Fluorescein fundus angiography revealed a retinal nonperfusion area only in the left eye, and panretinal photocoagulation was subsequently performed. Conclusion: Our findings show that the reason for the left-right difference in DR was attributed to the more severe choroidal coloboma and retinal thinning in the patient's right eye compared to his left eye, thus reducing oxygen demand, as is also seen in eyes with severe myopia.

\section{KARGER}

Tsunehiko Ikeda, MD

Department of Ophthalmology, Osaka Medical College 2-7 Daigaku-machi

Takatsuki, Osaka 569-8686 (Japan)

E-Mail tikeda@poh.osaka-med.ac.jp 
Moriya et al.: A Case of Uveal Colobomas Showing Marked Left-Right Difference in Diabetic Retinopathy

\section{Introduction}

Congenital uveal colobomas, including inferior iris and choroidal colobomas, are congenital anomalies associated with microcornea and microphthalmia and often show leftright differences (laterality) [1]. In this study, we present the case of a patient with uveal colobomas and diabetic retinopathy (DR) who showed a laterality of the choroidalcoloboma-related DR.

\section{Case Presentation}

The patient was a 59-year-old male with hyperglycemia (blood glucose, $363 \mathrm{mg} / \mathrm{dl}$; hemoglobin A1c, 13.4\%) who had previously been referred to an ophthalmologist for fundus examination by his primary physician. The examination revealed bilateral DR and uveal colobomas, so he was referred to our department for further evaluation and treatment. The patient's past medical history was unremarkable except for right-eye amblyopia and he had not previously been treated for diabetes.

Upon initial examination, the patient's visual acuity (VA) was $0.04 \times \mathrm{S}-6.00 \mathrm{D}=\mathrm{C}-1.50 \mathrm{D} A$ $\times 80$ in his right eye and $0.4 \times \mathrm{S}-1.75 \mathrm{D}=\mathrm{C}-0.50 \mathrm{D} \mathrm{A} \times 150$ in his left eye. His intraocular pres-

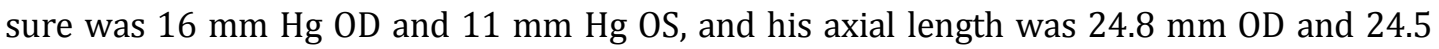
$\mathrm{mm}$ OS. Slit-lamp examination showed mild-to-moderate nuclear cataracts, and inferior iris colobomas in the optic media were observed in both eyes. Right-eye funduscopy showed an extensive inferior choroidal coloboma that included the optic disc and macula. The fundus showed a tigroid pattern due to myopia, yet presented almost no changes of DR (fig. 1a). Left-eye funduscopy also showed a choroidal coloboma, yet without involvement of the macula. The choroidal coloboma was seen in two areas, both inferiorly and temporoinferiorly near the optic disc.

The fundus also of the left eye showed multiple hemorrhages and marked hard exudates in the macula (fig. 1b). Optical coherence tomography (OCT) imaging of the right eye showed retinal thinning and inferior choroidal coloboma (fig. 2a). OCT of the left eye revealed macular hard exudates and temporal retinal edema, but without retinal thinning (fig. 2b). Fluorescein fundus angiography showed no areas of nonperfusion in the right retina (fig. 3a). In the left retina, except in the inferior choroidal colobomatous area, there were nonperfused areas in the circumferential periphery and contrast leakage over time (fig. 3b). Examination of the vitreous by slit-lamp microscopy with a front lens showed no posterior vitreous detachment (PVD) in either eye.

A retinal nonperfusion area was seen only in the patient's left eye, and panretinal photocoagulation was performed in three sessions. In addition, triamcinolone acetonide was injected under the Tenon's capsule for the macular edema in that eye. The hard exudates in the fovea and macular edema in that eye then gradually decreased, and VA was improved $(0.7 p$ $\times \mathrm{S}-1.00 \mathrm{D}=\mathrm{C}-1.00 \mathrm{D} \mathrm{A} \times 70$ ) by 8 months after the initiation of treatment. Carotid ultrasonography was also performed during this time to examine possible left-right differences in internal carotid artery (ICA) stenosis. However, no obvious left-right differences were evident. 
Moriya et al.: A Case of Uveal Colobomas Showing Marked Left-Right Difference in Diabetic Retinopathy

\section{Discussion}

Uveal colobomas are congenital anomalies that are usually caused by incomplete closure of embryonic fissures $[2,3]$. Uveal colobomas typically occur in the inferior part of the eye, including the choroid, ciliary body, and iris, and left-right differences (laterality) are often apparent. Colobomas are frequently associated with microcornea and microphthalmia, yet other ocular complications, including nystagmus, lens subluxation, cataracts, glaucoma, and retinal detachment, may occur. Systemic complications such as mental retardation, congenital heart disease, and hearing impairment have also been reported. Eyes with a coloboma are often amblyopic from birth, and VA is poor when the choroidal coloboma extends into the macula.

DR is a vascular complication associated with diabetes as a systemic metabolic disease. DR usually develops and progresses in both eyes, but distinct left-right differences (laterality) in DR may occur. The causes of left-right differences in DR can be broadly divided into local ocular factors and systemic factors. Local ocular factors that may inhibit the progression of DR include myopia, PVD, and optic nerve atrophy. Systemic factors that may worsen DR include circulatory disorders such as ICA stenosis and ophthalmic artery stenosis [4-6].

Retinal thinning in myopic eyes decreases the oxygen demands of the retina, thus reducing the risk of ischemic changes due to DR. This is particularly true in eyes with myopic chorioretinal atrophy in which progression to proliferative DR is usually rare. The vitreous is also involved in the progression of DR, and since retinal neovascularization usually develops along the edges of the posterior precortical vitreous pockets [7], these changes are less likely in patients with complete PVD. Progression of DR is also less likely in patients with glaucoma, central retinal artery occlusion, and optic disc atrophy associated with optical canal fractures. This is due to thinning of the inner retinal layer, which mainly comprises the ganglion cell layer, so retinal oxygen demand is also reduced.

ICA and ophthalmic artery stenosis are frequent systemic causes of left-right eye differences in DR cases. This is because unilateral ICA or ophthalmic artery stenosis can decrease blood circulation to the entire retina. These systemic conditions can be diagnosed using carotid ultrasound and a Doppler study.

Although the patient described in this present study showed differences in refraction between his left and right eyes, axial length was approximately the same bilaterally. Examination of the vitreous by slit-lamp microscopy with a front lens showed no evidence of PVD in either eye. In addition, carotid artery ultrasound revealed no stenosis on either side. Therefore, we theorize that the left-right differences in DR in our patient were related to the severity of the choroidal coloboma.

The mechanism of choroidal coloboma as an inhibitory factor in DR may involve the following: (1) lack of a nutrient supply from the choroid, despite the presence of retinal tissue, in the choroidal colobomatous area; (2) atrophy of the outer retinal layer, and (3) for the whole eye, similarly to severe myopia or optic nerve atrophy, a relative decrease in retinal oxygen demand [8]. Our patient showed extensive choroidal coloboma of the right eye involving the optic nerve and macular region. Hence, retinal development, other than in the choroidal colobomatous area, may also have been poor compared to the left eye. Indeed, OCT of the patient's right eye revealed retinal thinning in areas other than the choroidal coloboma. Perhaps because of the large choroidal coloboma, less blood supply was required in this area, and an environment may have developed in which more blood was supplied to the ophthalmoscopically healthy retina.

DR associated with uveal colobomas has seldom been reported. In a study about ocular complications involving 5 cases of uveal colobomas, Steahly et al. [9] reported 1 patient with 
Moriya et al.: A Case of Uveal Colobomas Showing Marked Left-Right Difference in Diabetic Retinopathy

DR, yet detailed information was not provided. Cellini et al. [10] described a patient with type 2 diabetes and hypertension who had DR and so-called changes of pseudodoubling of the optic disc, with the pseudodoubling possibly resulting from a choroidal coloboma. In any case, choroidal colobomas can affect retinal development and cause relative dysplasia of the retina, thus making DR less likely. However, the possibility of DR associated with mild choroidal coloboma, as in the left eye of the case presented in this current study, should also be kept in mind. These types of patients require careful follow-up evaluation.

\section{Statement of Ethics}

The authors have no ethical conflicts to disclose.

\section{Disclosure Statement}

The authors have no conflicts of interest to disclose.

\section{References}

1 Uhumwangho OM, Jalali S: Chorioretinal coloboma in a paediatric population. Eye 2014;28:728-733.

-2 Pagon RA: Ocular coloboma. Surv Ophthalmol 1981;25:223-236.

-3 Onwochei BC, Simon JW, Bateman JB, Couture KC, Mir E: Ocular colobomata. Surv Ophthalmol 2000;45:175194.

-4 Duker JS, Brown GC, Bosley TM, Colt CA, Reber R: Asymmetric proliferative diabetic retinopathy and carotid artery disease. Ophthalmology 1990;97:869-874.

5 El Hindy N, Ong JM: Asymmetric diabetic retinopathy. J Diabetes 2010;2:125-126.

6 Iino K, Yoshinari M, Kaku K, Yamamoto M, Sato Y, Kodama T, Iwase M, Fujishima M: Prospective study of asymmetric retinopathy as a predictor of brain infarction in diabetes mellitus. Diabetes Care 1993;16:14051406.

7 Kishi S, Shimizu K: Clinical manifestations of posterior precortical vitreous pocket in proliferative diabetic retinopathy. Ophthalmology 1993;100:225-229.

-8 Lingam G: Pattern of blood vessels in eyes with coloboma. Indian J Ophthalmol 2013;61:743-748.

-9 Steahly LP: Retinochoroidal coloboma: varieties of clinical presentations. Ann Ophthalmol 1990;22:9-14.

$\checkmark 10$ Cellini M, Alessandrini A, Bernabini B, Merlo D: Pseudodoubling of the optic disc: a colour Doppler imaging study. Ophthalmologica 2003;217:370-372. 
Case Reports in

Ophthalmology
Case Rep Ophthalmol 2016;7:167-173

\begin{tabular}{l|l}
\hline DOI: $10.1159 / 000445248$ & (C) 2016 The Author(s). Published by S. Karger AG, Basel
\end{tabular} www.karger.com/cop

Moriya et al.: A Case of Uveal Colobomas Showing Marked Left-Right Difference in Diabetic Retinopathy
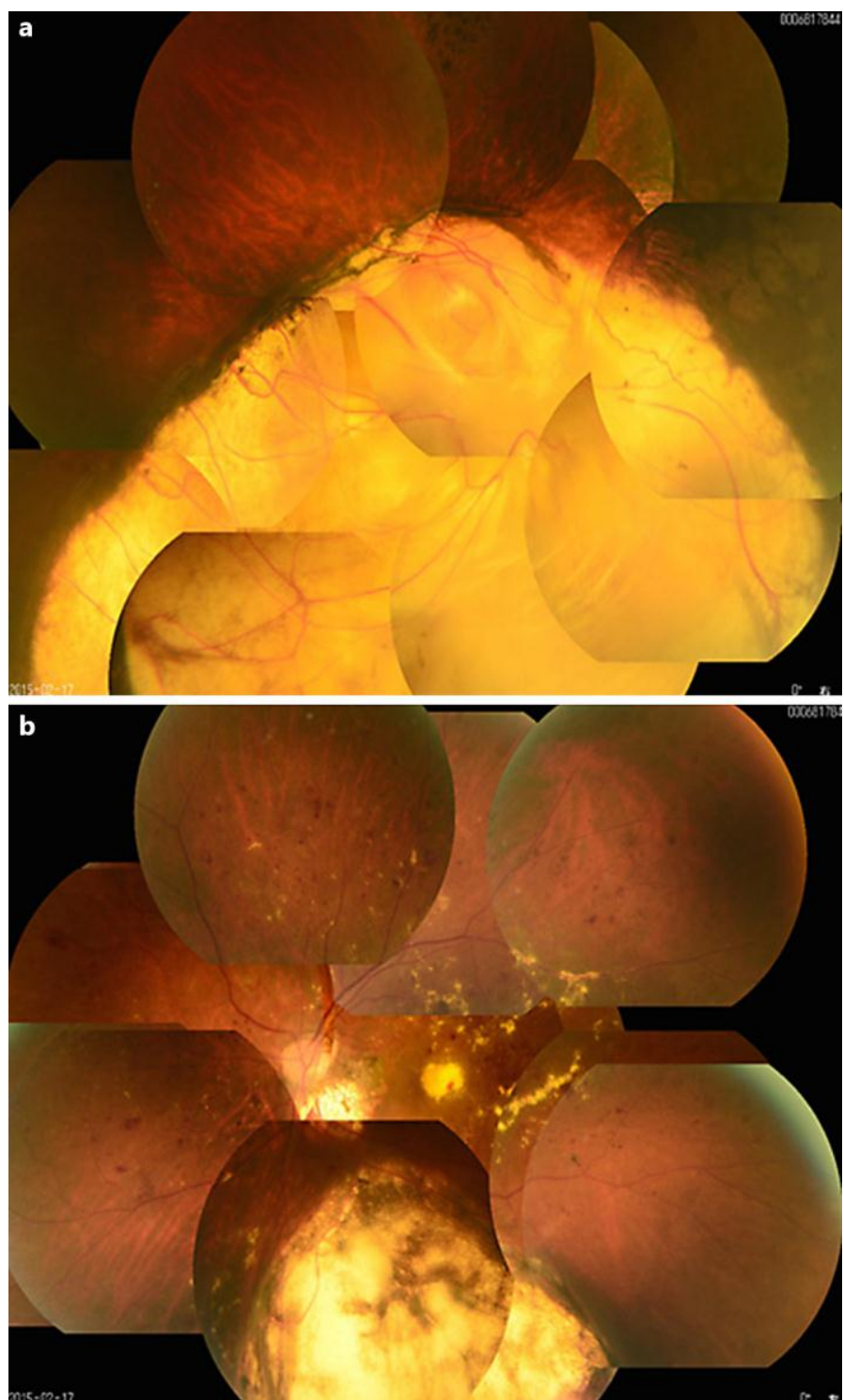

Fig. 1. Fundus photography of the right and left eyes. Extensive choroidal coloboma is seen in the right eye (a), markedly different from the left eye (b). 


\section{Case Reports in \\ Ophthalmology}

\begin{tabular}{l|l}
\hline Case Rep Ophthalmol 2016;7:167-173 \\
\hline DOI: 10.1159/000445248 & $\begin{array}{l}\text { @ 2016 The Author(s). Published by S. Karger AG, Basel } \\
\text { www.karger.com/cop }\end{array}$ \\
\hline
\end{tabular}

Moriya et al.: A Case of Uveal Colobomas Showing Marked Left-Right Difference in Diabetic Retinopathy
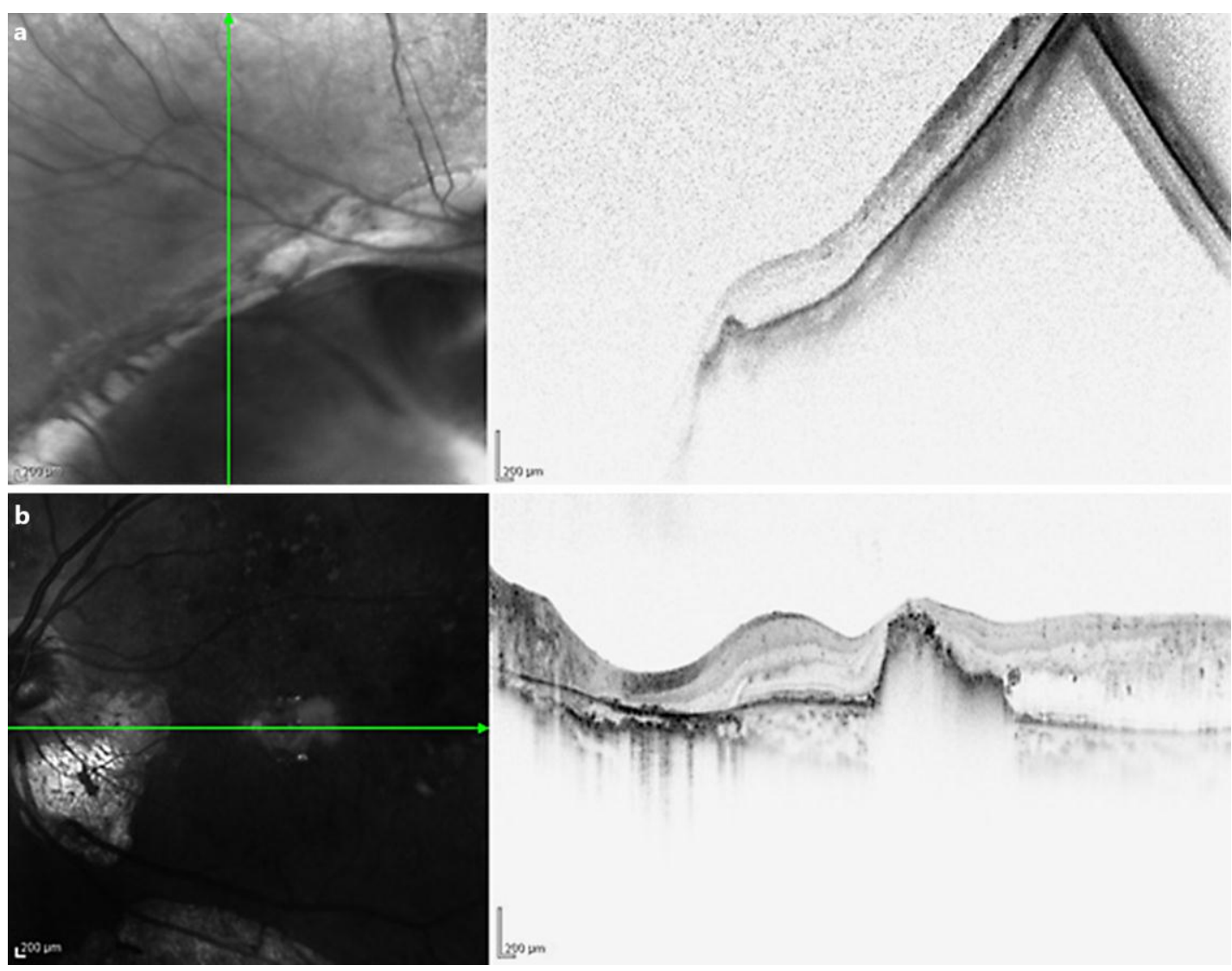

Fig. 2. OCT of the right and left eyes. The right eye (a) shows retinal thinning. The retina is thicker in the left eye (b) than in the right eye, and foveal hard exudates are present. 
Case Reports in

Ophthalmology
Case Rep Ophthalmol 2016;7:167-173

\begin{tabular}{l|l}
\hline DOI: $10.1159 / 000445248$ & C 2016 The Author(s). Published by S. Karger AG, Basel
\end{tabular} www.karger.com/cop

Moriya et al.: A Case of Uveal Colobomas Showing Marked Left-Right Difference in Diabetic Retinopathy
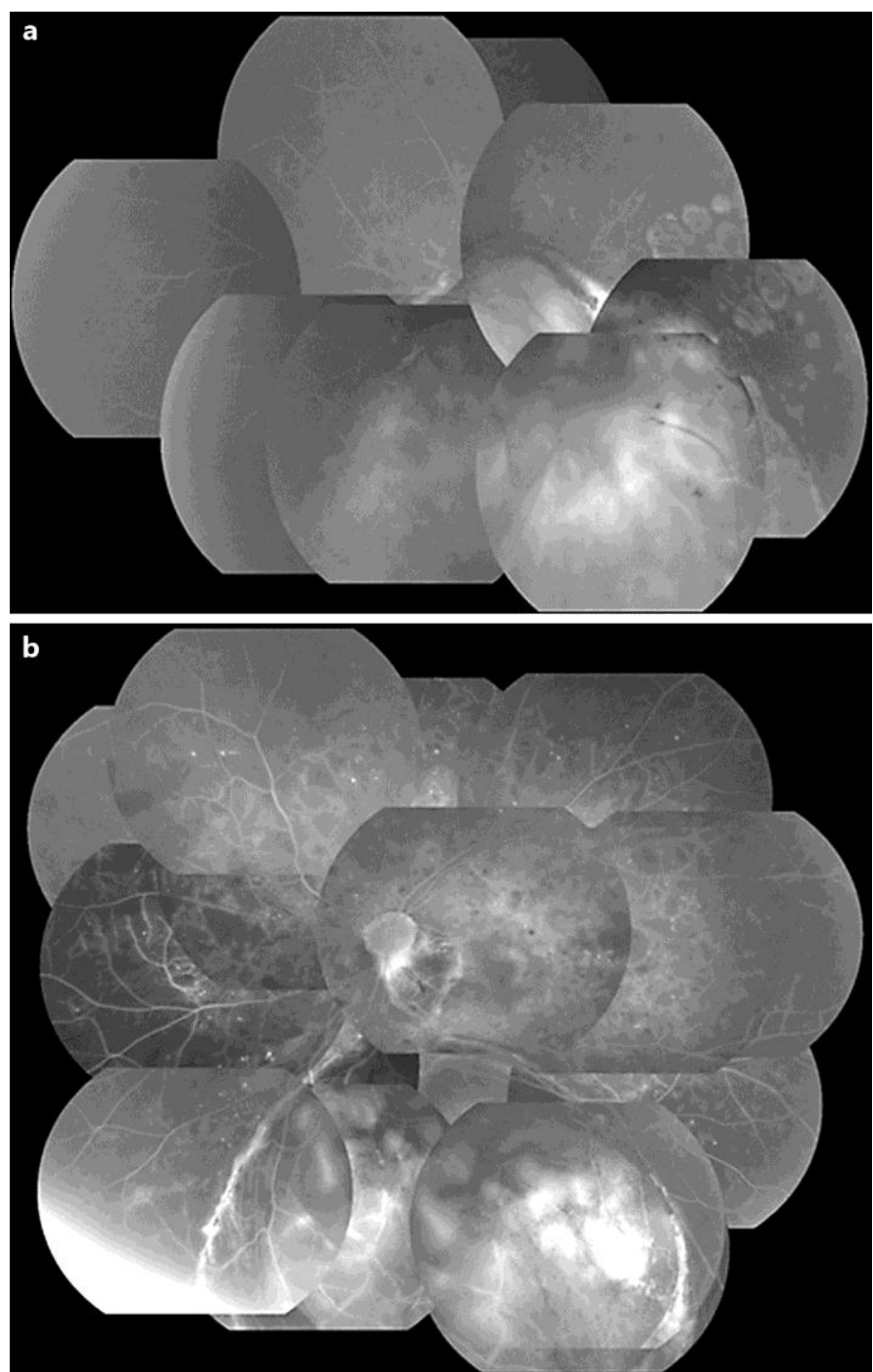

Fig. 3. Fluorescein fundus angiography of the right and left eyes. a The right eye shows no changes of DR b The left eye displays a wide area of retinal nonperfusion. 